\title{
Using rainfall thresholds and ensemble precipitation forecasts to issue and improve urban inundation alerts
}

\author{
Tsun-Hua Yang ${ }^{1}$, Gong-Do Hwang ${ }^{1,2}$, Chin-Cheng Tsai ${ }^{1}$, and Jui-Yi Ho ${ }^{1}$ \\ ${ }^{1}$ Taiwan Typhoon and Flood Research Institute (TTFRI), National Applied Research Laboratories (NARLabs), \\ Taipei, 10093, Taiwan \\ ${ }^{2}$ Department of Atmospheric Science, National Taiwan University, Taipei, 10617, Taiwan \\ Correspondence to: Tsun-Hua Yang (tshyang@ narlabs.org.tw)
}

Received: 8 July 2016 - Published in Hydrol. Earth Syst. Sci. Discuss.: 29 July 2016

Revised: 8 November 2016 - Accepted: 16 November 2016 - Published: 30 November 2016

\begin{abstract}
Urban inundation forecasting with extended lead times is useful in saving lives and property. This study proposes the integration of rainfall thresholds and ensemble precipitation forecasts to provide probabilistic urban inundation forecasts. Utilization of ensemble precipitation forecasts can extend forecast lead times to $72 \mathrm{~h}$, predicting peak flows and to allow response agencies to take necessary preparatory measures. However, ensemble precipitation forecasting is time- and resource-intensive. Using rainfall thresholds to estimate urban areas' inundation risk can decrease this complexity and save computation time. This study evaluated the performance of this system using 352 townships in Taiwan and seven typhoons during the period 2013-2015. The levels of forecast probability needed to issue inundation alerts were addressed because ensemble forecasts are probability based. This study applied six levels of forecast probability and evaluated their performance using five measures. The results showed that this forecasting system performed better before a typhoon made landfall. Geography had a strong impact at the start of the numerical weather modeling, resulting in the underestimation of rainfall forecasts. Regardless of this finding, the inundation forecast performance was highly contingent on the rainfall forecast skill. This study then tested a hybrid approach of on-site observations and rainfall forecasts to decrease the influence of numerical weather predictions and improve the forecast performance. The results of this combined system showed that forecasts with a $24 \mathrm{~h}$ lead time improved significantly. These findings and the hybrid approach can be applied to other hydrometeorological early warning systems to improve hazard-related forecasts.
\end{abstract}

\section{Introduction}

Flooding is one of the most destructive disasters in the world and results in enormous losses of life and property annually (Gruntfest and Handmer, 2001; Barredo, 2009; Hallegatte et al., 2013; Sampson et al., 2015). Global flood risk is likely to increase under climate change; as a result, numerous adaption strategies should be considered (Hirabayashi et al., 2013). Establishing an early flood warning system to reduce disaster losses is the most cost-effective solution of all of the structural and non-structural measures studied (Alfieri et al., 2012; Hallegatte, 2012). Several flood warning systems have been developed and implemented in response to floods (Pappenberger et al., 2005; Thielen et al., 2009; López-Trujillo, 2010; De Kleermaeker et al., 2012; Doong et al., 2012).

Various approaches are used to simulate flooding based on the available rainfall data. Complex models such as the one- or two-dimensional Saint-Venant equations better describe flow behaviors and provide detailed spatial information as part of their flood forecasts (e.g., Nguyen et al., 2015; Huthoff et al., 2015). However, the high computation costs and substantial data requirements involved in solving these detailed models limit the application of these models during an emergency response or real-time forecast. Therefore, a variety of alternatives, such as simplified equation-based systems, data-driven models, and rainfall threshold-based approaches, have been developed to improve the computing efficiency of the models. 
- Simplified equation-based systems (e.g., Cirbus and Podhoranyi, 2013; Liu et al., 2015; Shao et al., 2015) use simplified equations, such as Manning's equation, to describe water spreading, thereby improving the calculation efficiency of the forecasting models. However, the data required, including digital elevation models (DEMs) and surface roughness, are sometimes difficult to collect. As a result, data preparedness is still a practical concern for the abovementioned models.

- Data-driven models are based on computational intelligence or machines. Flood forecasting is just one of the applications of these models (e.g., Chang et al., 2010; Lin et al., 2013). As indicated by the name, the quality and quantity of data used in the model have a considerable impact on the performance of data-driven models. To collect accurate flood inundation data is a challenge in itself. In addition, the performance of data-driven models deteriorates as forecast time increases (e.g., Lin and Jhong, 2015; Badrzadeh et al., 2015). Data-driven models also cannot provide forecasts with longer lead times.

- A rainfall threshold approach is commonly applied to evaluate landslide risk (e.g., Crosta and Frattini, 2003; Guzzetti et al., 2007; Posner and Georgakakos, 2015). Meteorological organizations generally issue flood forecasts/warnings if a critical value - namely, a rainfall threshold - is exceeded by the observed or predicted rainfall (Martina et al., 2006). Several operational meteorological agencies throughout the world issue warnings based on Flash Flood Guidance (FFG) values (Gourley et al., 2014). The US National Weather Service (NWS) developed FFG values for flash flooding (Carpenter et al., 1999). Based on these values, floods are predicted, and flood warnings are issued Georgakakos $(2005,2006)$ studied operational flash flood warning systems based on FFG and provided analytical results. These studies found that an FFG threshold is likely to produce a high probability of detection in regions where flash floods are frequent. The European Flood Awareness System (EFAS) uses numerical weather predictions and the Enhanced Runoff Index based on Climatology, which is based on simulated climatology, an FFG-related concept, to provide flash flood warnings (Raynaud et al., 2015). In countries such as Kenya and Haiti that do not have enough well-trained operators and resources to set up an efficient flood warning system, the approach is a viable alternative that allows for the mitigation of flood damage (Georgakakos et al., 2013; Shamir et al., 2013; Hoedjes et al., 2014). The rainfall threshold approach has proven successful in identifying a number of flash floods across Europe (Alfieri et al., 2014). Although it should not be considered a substitute for complex hydro-meteorological models because of its simplicity, using a rainfall threshold approach to develop a flood warning system can be an immediately useful tool for a variety of decision makers interested in early warnings and flash floods (Martina et al., 2006). Only a few studies (Jang, 2015; Wu et al., 2015) have applied rainfall thresholds to evaluate urban inundation risk. The present study represents the first of its kind to use the rainfall threshold approach and quantitative precipitation forecasts (QPFs) to evaluate inundation risk in Taiwan. By directly comparing QPFs with critical rainfall thresholds, this study aims to propose an early warning system that provides forecasts, allows for the possibility of issuing urban inundation warnings and gives response agencies enough lead time to implement emergency preparedness plans.

A flood warning system that uses QPFs as the rainfall input could increase the forecasting horizon from a few hours to a few days (Pappenberger et al., 2005; Shi et al., 2015). Georgakakos (2005) concluded that the dominant source of uncertainty in applying a rainfall thresholds approach to evaluate flood risk is precipitation. The uncertainty in forecasted rainfall values is generally higher than that for observed rainfall data. Nevertheless, to extend the forecast lead time, operational and research flood forecasting systems around the world are increasingly moving toward using QPFs to provide early warnings (Cloke and Pappenberger, 2009). Martina et al. (2006) discussed the possibility of providing flood warnings at given river reaches by directly comparing the QPF to a critical rainfall threshold value. Regardless of the forecasts' uncertainty, considering which probabilistic forecast levels should be used to issue inundation alerts or take actions is a challenging topic. Higher levels of probabilistic forecasts usually give the practitioner more confidence in the results. Dale et al. (2014) proposed a risk-based decisionsupport framework that could be easily applied in an operational flood forecast and early warning context. Other studies have also discussed the selection of appropriate probabilistic forecasts in terms of the economic and practical consequences of taking action (Coughlan de Perez et al., 2015, 2016). Therefore, the present study evaluates the system's performance in terms of different levels of forecast probability. In addition, this study proposes a data assimilation technique that uses real-time observations to decrease the uncertainty from rainfall forecasts and increases the $24 \mathrm{~h}$ forecast accuracy.

\section{System development}

The proposed inundation early warning system integrates ensemble precipitation forecasts, rainfall thresholds, and a realtime data assimilation technique to assess the possibility of issuing inundation alerts. Figure 1 shows the system's operational process during a typhoon event. The forecast results are intended to be provided to practitioners through a web 


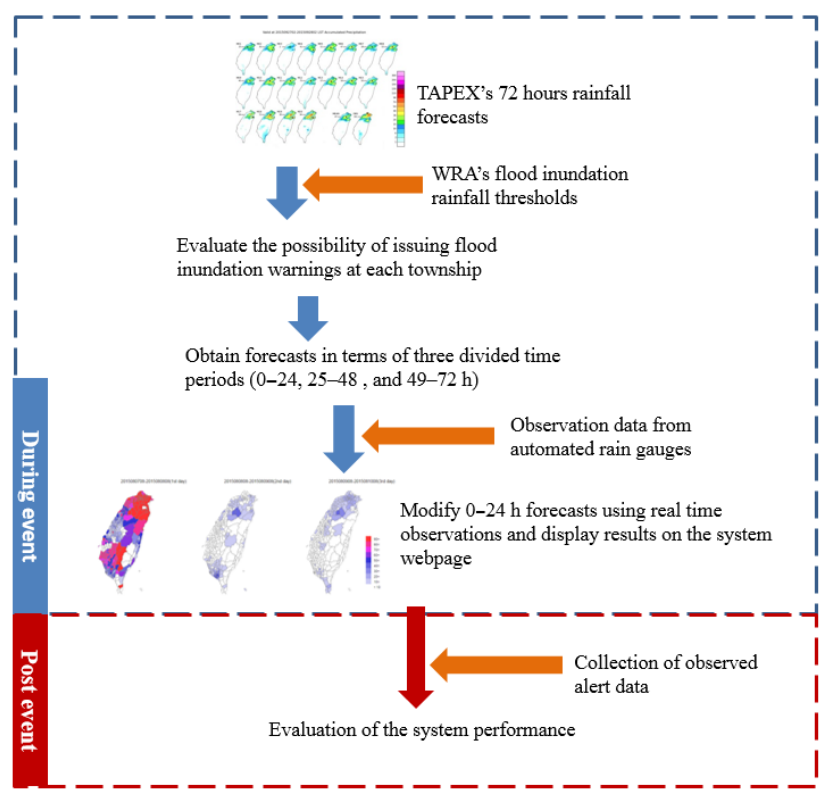

Figure 1. The operational flow chart for the proposed urban inundation early warning system.

page. Due to a limitation in the computing resources and data retrieval tools available, the system generates a forecast every $6 \mathrm{~h}$ and updates the results on the web page. The details of each component in the system are as follows.

\subsection{Ensemble precipitation forecasts for system input}

This study used rainfall forecasts from a precipitation ensemble forecast experiment, namely, the Taiwan cooperative precipitation ensemble forecast experiment (TAPEX). TAPEX is a collective effort among academic institutes and government agencies such as National Taiwan University (NTU), National Central University (NCU), National Taiwan Normal University (NTNU), Chinese Culture University (CCU), the Central Weather Bureau (CWB), the National Center for High-Performance Computing (NCHC), the Taiwan Typhoon and Flood Research Institute (TTFRI), and the National Science and Technology Center for Disaster Reduction (NCDR). The experiment began in 2010 and was the first attempt to design a high-resolution numerical ensemble weather model in Taiwan. The experiment collects worldwide observation data, including temperature, wind, surface pressure, and relative humidity, from satellites, atmospheric sounding devices, buoys, aviation routine weather reports, ships, and other available sources (e.g., Hsiao et al., 2012, 2013). TAPEX uses the outputs from the Global Forecast System (GFS) produced by the National Centers for Environment Prediction (NCEP), along with observation data, as the initial and boundary conditions for its forecasts. Various model physics schemes and data assimilation strategies are used to perturb the numerical weather models and create dif-

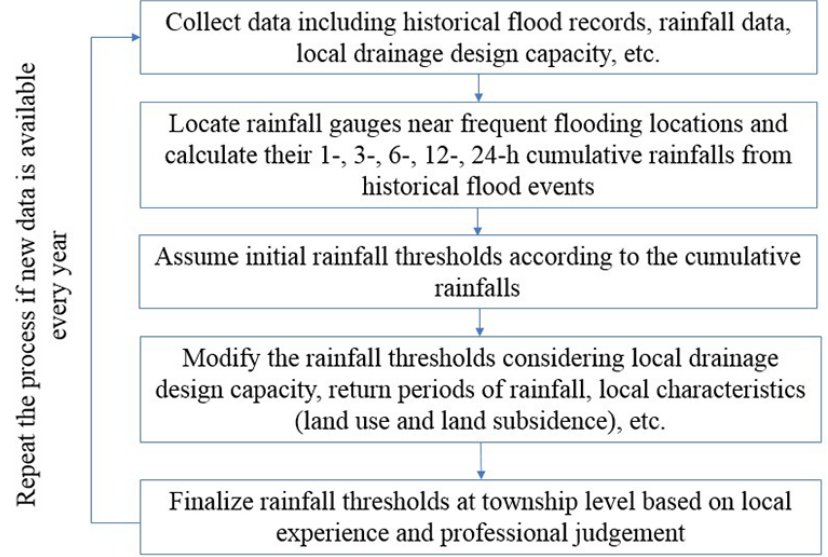

Figure 2. The identification process of rainfall thresholds (modified from Wu and Wang, 2009).

ferentiated ensemble members. To date, 20 ensemble members and 4 different regional models (AWR-WRF, HWRF, MM5 and CreSS) have been established for precipitation forecasting. The experiment aims to provide 24,48 , and $72 \mathrm{~h}$ typhoon rainfall forecasts and generates four runs per day at a $5 \mathrm{~km}$ spatial resolution. TAPEX's rainfall forecasts can extend the inundation forecast lead time to $72 \mathrm{~h}$, which exceeds the average rainfall-runoff concentration time and the lag between observed peak participation and flooding in Taiwan. This lead time is thus considered sufficient for decisionmaking processes to be implemented prior to inundation.

\subsection{Rainfall threshold for urban inundation alerts}

Coughlan de Perez et al. (2016) defined the danger level of flooding as the 95th percentile of a flood model's forecasts at a lead time of $0 \mathrm{~h}$. The present study considered rainfall thresholds as danger levels related to the likelihood of urban inundation. In Taiwan, the Water Resources Agency (WRA) has developed rainfall thresholds for all townships (Wu and Wang, 2009). Figure 2 shows the WRA's identification process for rainfall thresholds at the township level. It starts by collecting historical flood records that show when and where a flood occurred. The initial rainfall thresholds can then be estimated by determining the cumulative rainfall amounts at nearby rain gauges. The finalized rainfall thresholds for different townships are based on further investigations of local drainage capacity, local characteristics (e.g., land subsidence), and the professional judgement of local experts. The WRA reviews the rainfall thresholds every year once the new records are available. Inundation alerts are issued when observed rainfall meets or exceeds a given rainfall threshold. Local governments and civil agencies take necessary measures such as evacuating residents and deploying dewatering pumps based on the alerts. Given the historical record, the WRA assumes that inundations are directly related to accumulated rainfall and use a regression analysis to identify a 
two-level alarm for five duration periods. The five duration periods are $1,3,6,12$, and $24 \mathrm{~h}$; a total of 10 rainfall thresholds are used to issue urban inundation alerts. The two levels of alarms are defined as follows:

- First-level alert: If the rain continues, the roads and villages subject to a high risk of flooding in the alerted townships may flood.

- Second-level alert: If the rain continues, the roads and villages subject to a high risk of flooding in the alerted townships will flood in the next $3 \mathrm{~h}$.

The WRA has associated different rain gauges with different townships and issues warnings by comparing the observations with the associated rain gauges. The rainfall thresholds for the first and second alerts are different. There is a $3 \mathrm{~h}$ lead time before flooding if the accumulated rainfall reaches the second-level alert. The first-level alert is at an immediate risk of flooding. The WRA identified the rainfall thresholds of the second-level alerts for the purpose of precaution so that the responding authorities have time to take action. An inundation alert is issued if any of the rainfall thresholds is met by the observed rainfall. Wu (2013) compared the alerts to collected inundation records in 2012 and 2013 and concluded that the forecast accuracy rate is above $60 \%$. As the only rainfall threshold approach used to issue inundation alerts in Taiwan, it has proven its applicability in predicting flood inundation. This study used the rainfall thresholds of the second-level alerts to develop an early flood warning system.

\subsection{Inundation risk evaluation and a data assimilation technique to modify the forecasts}

In practice, the WRA issues inundation alerts when the cumulative rainfall exceeds the rainfall threshold at time $T$ (Fig. 3). However, WRA compares real-time precipitation observations to the rainfall thresholds, and thus the lead time is usually not long enough to allow communities to implement emergency preparedness measures. This study proposes a practical early warning system that compares cumulative projected rainfall instead of observed rainfall to provide probabilistic urban inundation forecasts. The system uses TAPEX's forecasted rainfall to extend the model's lead time to $72 \mathrm{~h}$. Figure 4 shows the forecast length during a realtime operation. TAPEX uses available observations at $t-6$ as its model's initial conditions, and its numerical weather model computation process took $6 \mathrm{~h}$ to produce rainfall forecasts from $t$ to $t+72 \mathrm{~h} ; 352$ Taiwanese townships were used in this study to evaluate the proposed system's performance. Equations (1) and (2) were used to calculate the probability of inundation in any given township; the forecasts were displayed over three distinct time periods (1-24, 25-48, and 49-72 h). A rolling window approach was applied to estimate the probability of issuing an inundation alert: each hour

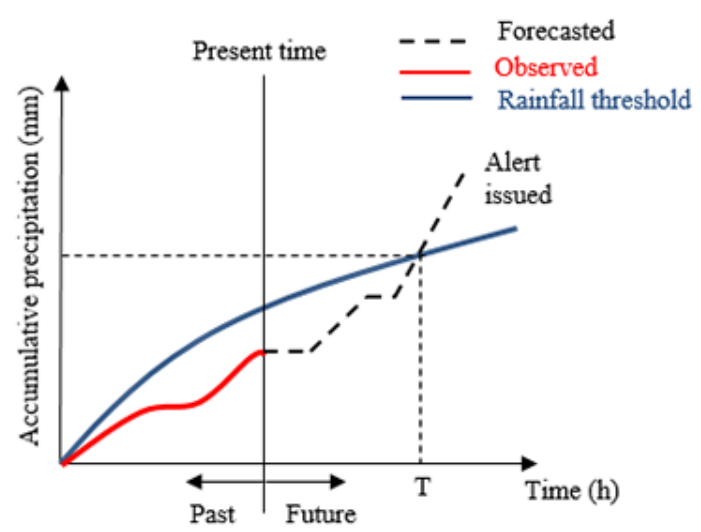

Figure 3. WRA issues an inundation alert when observed rainfalls meet or exceed any given rainfall thresholds (modified from Martina et al., 2006).

of the forecasting period was considered an evaluation end point, and the cumulative rainfall was calculated for the different durations.

$f_{i}=\left\{\begin{array}{ll}1 & \text { if } \mathrm{PF}_{i, \text { accu }} \geq \mathrm{PT}_{\text {dur }} \\ 0 & \text { if } \mathrm{PF}_{i, \text { accu }}<\mathrm{PT}_{\text {dur }}\end{array}\right.$ dur $=\{1,3,6,12,24 \mathrm{~h}\}$,

where $\mathrm{PF}_{i, \text { accu }}$ is the cumulative forecasted rainfall of the $i$ th ensemble member in TAPEX. $\mathrm{PT}_{\text {dur }}$ represents cumulative rainfall thresholds for the different durations (dur) $(1,3,6$, 12 , and $24 \mathrm{~h})$. An inundation occurred $\left(f_{i}=1\right)$ if the cumulative forecasted rainfall exceeded any of these thresholds.

$\operatorname{Pr}=\frac{1}{N} \sum_{i=1}^{N} f_{i} \times 100, \quad N=1,2, \ldots, 20$

There are 20 ensemble members $(N=20)$ in TAPEX. Equation (2) sums the $f_{i}$ values to obtain a probability (Pr), which represents the inundation risk for any given township. Each township's inundation risk can be obtained by repeating the above steps and comparing the results to TAPEX's $72 \mathrm{~h}$ rainfall forecasts. Three separate time periods (1-24, 25-48, and 49-72 h) illustrate the township's future inundation risk.

The accuracy of the rainfall forecasts has a considerable impact on the flood inundation forecasts. McBride and Ebert (2000) revealed that most numerical global models over-predicted and slightly under-predicted the rainfall frequency of various thresholds in Australia in summer and winter, respectively. These authors used a bias score (bias) to address the over- or under-estimation issue. A prediction is underestimated if the bias is less than 1.0. McBride and Elbert (2000) found that the biases of most numerical models were less than 1.0 for rainfall thresholds greater than $20 \mathrm{~mm}_{\text {day }^{-1}}$. The TAPEX under-predicted the rainfall frequencies during a rainfall event greater than $100 \mathrm{~mm} \mathrm{day}^{-1}$ according to the forecast results in 2016. The 


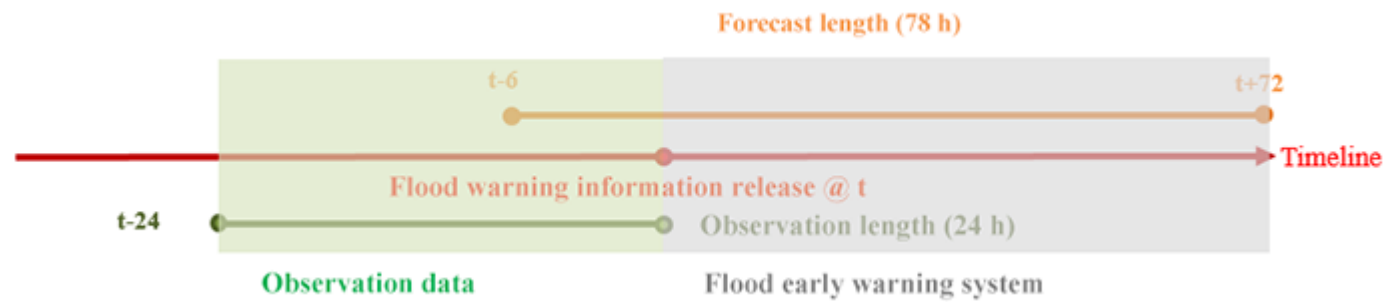

Figure 4. A combination of real-time rainfall observations and forecasts to improve 1 to $24 \mathrm{~h}$ inundation forecasts.

biases were 0.49 and 0.12 for rainfall thresholds of 100 and $350 \mathrm{~mm} \mathrm{day}^{-1}$, respectively. The complexity of the earthatmosphere system and associated physical interactions adds uncertainty to the ensemble rainfall forecasts. However, this is beyond the scope of this study. The purpose of this study is to provide flood warnings by adopting the existing uncertainties in numerical weather forecasts and to improve the forecasts by using a data assimilation technique that uses realtime rainfall observations. This study used the technique to modify the $24 \mathrm{~h}$ urban inundation forecast performance. Figure 4 illustrates the combination of observations and forecasts used in the forecasting process. This study utilized five rainfall thresholds to represent different rainfall durations. However, these five thresholds could not be applied to evaluate the inundation risk at every hour within the first $24 \mathrm{~h}$ forecast. For example, only one rainfall threshold covers the $1 \mathrm{~h}$ period, which can be considered time $t$ in Fig. 3; however, there is a lack of forecasts for $t-1$ and the preceding hours. When $t=t+2$, only rainfall thresholds for 1 and $3 \mathrm{~h}$ can be adopted. This shortcoming results in the underestimation of inundation forecasts. Given the above assumption, all five duration periods are applicable after the 25th hour. This study proposes a data assimilation technique using observed rainfall data to address the absence of rainfall forecasts. It applies available observation data from $t-24$ to $t-1$ prior to issuing inundation forecasts at $t$ (Fig. 4). Figure 2 combines the observation data (red line) and forecasts (dash line) with all rainfall thresholds (solid blue line). Alerts are issued if the combination exceeds the rainfall threshold at any given duration. In other words, the inundation forecast is improved within the first $24 \mathrm{~h}$.

\section{Study area and data}

\subsection{Study area}

Taiwan has an area of approximately $36000 \mathrm{~km}^{2}$, and approximately $70 \%$ of the island is covered by mountains. A mountain range runs through the center of the island from north to south and forms a ridge dividing the east- and westbound rivers. The rest of the island is composed of alluvial plains below $100 \mathrm{~m}$ in elevation. Ninety percent of the population lives on these alluvial plains. The distance from the mountaintops to the sea is very short, less than $70 \mathrm{~km}$ on average. Most of the riverbed slopes exceed $1 / 100$ in the upstream reaches and are between $1 / 200$ and $1 / 500$ in the downstream reaches, which results in average rainfall-runoff concentration times of between 6 and $72 \mathrm{~h}$ in the townships (Jang, 2015) and a lag time between observed peak precipitation and flooding of between 2 and $10 \mathrm{~h}$ (Jang et al., 2012) time and high density of the population in the plains areas further increase the damage caused by floods. Taiwan is one of the most disaster-prone countries in the world; thus, it has been selected as the study area here for the development of an urban inundation warning system.

\subsection{Observed inundation alerts}

Records such as the time of occurrence, depth, and extent of inundation are used to calibrate and validate early warning systems. Collecting accurate information is thus incredibly important. However, data collection during major floods is challenging. For example, identifying the occurrence time of an inundation is always an issue because of the lack of in situ monitoring devices. This study used urban inundation alerts issued by the WRA as a reference to evaluate the system's performance. The WRA issues alerts following the Common Alerting Protocol (CAP), which was first published by the OASIS Emergency Management Technical Committee in 2005 (OASIS Emergency Management Technical Committee, 2005). The WRA updates its alerts every $10 \mathrm{~min}$ and uploads the information to an open-source platform operated by the National Science and Technology Center for Disaster Reduction (Lee et al., 2014). The CAP data include observed flood warning information, such as the flood warning's location and duration. Information on seven typhoons, including Soulik (2013), Trami (2013), Matmo (2014), Fung-wong (2014), Linfa (2015), Soudelor (2015), and Dujuan (2015), was collected to evaluate the system's performance. Five of these typhoons made landfall and resulted in heavy rainfall and floods. For example, Soudelor dropped more than $1100 \mathrm{~mm}$ of precipitation within $24 \mathrm{~h}$ and had wind gusts of up to $66.1 \mathrm{~m} \mathrm{~s}^{-1}$ in northern Taiwan (i.e., Suao Township, Yilan County). Detailed information on these seven typhoons is listed in Table 1. The landfall time was identified when the eye of the typhoon made landfall. Of these typhoons, the eyes of Trami and Linfa did not 
Table 1. Information of seven typhoons during 2013-2015 used to evaluate the system performance.

\begin{tabular}{llll}
\hline Year & Name & Warning period (LST) & Landfall time (LST) \\
\hline 2013 & Soulik & $2013 / 07 / 1108: 30-2013 / 07 / 1323: 30$ & $2013 / 07 / 1303: 00$ \\
2013 & Trami & $2013 / 08 / 2011: 30-2013 / 08 / 2208: 30$ & $2013 / 08 / 21 / 18: 00^{*}$ \\
2014 & Matmo & $2014 / 07 / 2117: 30-2014 / 07 / 23 ~ 23: 30$ & $2014 / 07 / 2300: 10$ \\
2014 & Fung-wong & $2014 / 09 / 1908: 30-2014 / 09 / 2208: 30$ & $2014 / 09 / 2110: 00$ \\
2015 & Linfa & $2015 / 07 / 0608: 30-2015 / 07 / 0905: 30$ & $2015 / 07 / 0815: 00^{*}$ \\
2015 & Soudelor & $2015 / 08 / 0611: 30-2015 / 08 / 0908: 30$ & $2015 / 08 / 0804: 40$ \\
2015 & Dujuan & $2015 / 09 / 2708: 30-2015 / 09 / 2917: 30$ & $2015 / 09 / 2817: 40$ \\
\hline
\end{tabular}

* For typhoons that did not make landfall, this study defined the landfall time while the minimum observational station pressure was observed when typhoon was closest to Taiwan.

Table 2. Contingency table used for the system performance evaluation.

\begin{tabular}{|c|c|c|c|}
\hline & & \multicolumn{2}{|c|}{ CAP records from WRA } \\
\hline & & Issued & Not issued \\
\hline $\begin{array}{l}\text { Forecasted by the } \\
\text { proposed system }\end{array}$ & $\begin{array}{l}\text { Issued } \\
\text { Not issued }\end{array}$ & $\begin{array}{l}\text { Hit } \\
\text { Miss }\end{array}$ & $\begin{array}{l}\text { False alarm } \\
\text { No event }\end{array}$ \\
\hline
\end{tabular}

make landfall. For reference, this study selected the minimum observed atmospheric pressure at a weather station to define the time when these two typhoons were closest to Taiwan. The selected weather stations were the Taipei station for Trami and the Kaohsiung station for Linfa.

\section{Results and discussion}

This study relied on the contingency information shown in Table 2 to evaluate the performance of the proposed system. Hits and misses were associated with the observed records and determined based on whether the system's warning forecasts were consistent with the observations. A false alarm was associated with forecasts that did not correlate with observed data. "No event" was assigned to a township when neither the CAP records nor the model indicated flooding. Because floods are not frequent events, the no event (no flooding) scenario typically had a higher frequency than the other three fields. Different measures that have been broadly adopted by previous studies (e.g., Nguyen et al., 2015; Yang et al., 2015; Zhang et al., 2015) were used to evaluate the system's performance:

probability of detection $(\mathrm{POD})=\frac{\mathrm{Hit}}{\mathrm{Hit}+\text { Miss }}$,

false alarm ratio $($ FAR $)=\frac{\text { False alarm }}{\text { Hit }+ \text { false alarm }}$,

success ratio $(\mathrm{SR})=\frac{\text { Hit }}{\text { Hit }+ \text { false alarm }}$

threat score $(\mathrm{TS})=\frac{\text { Hit }}{\text { Hit }+ \text { Miss }+ \text { false alarm }}$.
Both POD and TS are sensitive to hits and range from 0 to 1. The only difference between these two values is that POD ignores false alarms and TS does not. POD has the ability to be artificially improved by the issuance of additional alarms, which would increase the number of hits. TS is also known as the critical success index (CSI) and usually results in poorer scores for rare events. SR and FAR are the success ratio and false alarm ratio, respectively. FAR is used in conjunction with POD. If FAR equals 0.5 or less, the performance is considered tolerable (Coughlan de Perez et al., 2016). The sum of SR and FAR equals 1, and both indices ignore misses. This study combined SR and FAR into one index (SR-FAR) that had a range from -1 to 1 . A positive value $(>0)$ for $S R-F A R$ was expected given that the likelihood of correct warnings is acceptable. Rare events such as floods result in extremely large numbers of no events, which could greatly affect the forecast results. In this study, a no event forecast can provide information to decision makers that allows them to allocate resources to those townships with a higher inundation risk. Equations (3)-(6) do not consider the "no event" scenario in their formulas. The accuracy (ACC) of the model, which is shown in Eq. (7) and is also called the proportion of correct forecasts (Wilks, 2005), is simple and intuitive, and it served as a valuable reference in this study.

Accuracy $(\mathrm{ACC})=$

$$
\text { Hit }+ \text { No event }
$$

$\overline{\mathrm{Hit}}+$ False alarm + Miss + No event

The next section presents the performance evaluation of the proposed system and then modifies the forecasting results using a hybrid of real-time observation and rainfall forecasts to improve the first $24 \mathrm{~h}$ inundation forecasts. This study used the time the typhoon made landfall as a reference point to define the evaluation period. The time needed to generate a rainfall forecast is $6 \mathrm{~h}$, noted as one date-time group (dtg). The evaluation period was plus-minus three dtg's $(18 \mathrm{~h})$ relative to the time at which a typhoon made landfall. For example, Table 1 shows that Trami made landfall at 18:00, $21 \mathrm{Au}-$ gust 2013. The landfall dtg is at 14:00, 21 August for 1-24 h; 14:00, 20 August for 25-48 h; and 14:00, 19 August for 49$72 \mathrm{~h}$. The $-1 \mathrm{dtg}$ is 08:00, 21 August for 1-24 h; 08:00, 20 


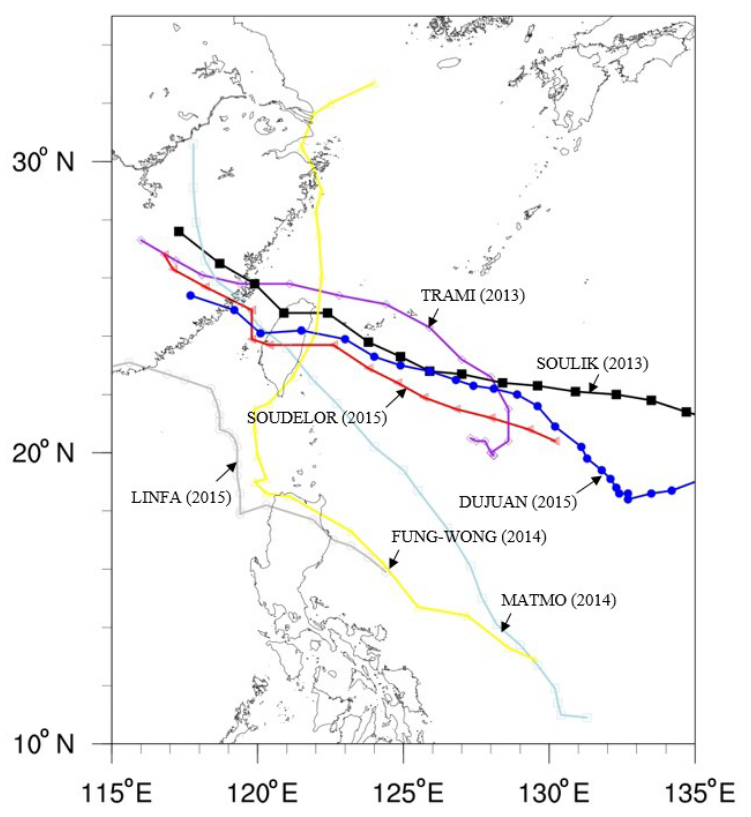

Figure 5. Location of the island of Taiwan, seven typhoons during 2013-2015, and their observed tracks.

August for 25-48 h; and 08:00, 19 August for 49-72 h. The average impact duration of a typhoon in Taiwan is $73.68 \mathrm{~h}$ (Huang et al., 2012). A typhoon has the most impact during the evaluation period (a total of $36 \mathrm{~h}$ ).

\subsection{Original forecast results without a data assimilation technique}

Both the typhoon tracks and geography affected the performance of the rainfall forecasts. Figure 5 shows the observed typhoon tracks, and Fig. 6 compares the forecasted and observed tracks for Soulik, Soudelor, and Matmo. The models of the first two typhoons were consistent with the observed tracks, while the third was not; as a result, the performance of rainfall forecasts during the first two typhoons exceeded that of the third typhoon. The causes of the track forecast errors are beyond the discussion of this study. Use of ensemble rainfall forecasts as inputs to produce flood warning forecasts should take into account uncertainties such as track and rainfall forecast errors in numerical weather predictions. Figures 7-9 show the differences between the observed and forecasted flood warnings without a data assimilation technique over three lead-time periods $(1-24,25-48$, and $49-72 \mathrm{~h}$ ). Tables $3-5$ summarize the average ACC, POD and SR-FAR results for different lead-time lengths during the evaluation period. The proposed system provides probabilistic forecasts. For example, $50 \%$ flood probability means that at least 10 out of 20 TAPEX members produced rainfall forecasts that met or exceeded the rainfall thresholds. The appropriate probability threshold that initiated response actions was discussed. Six probability thresholds $(10,30,50,70,80$,
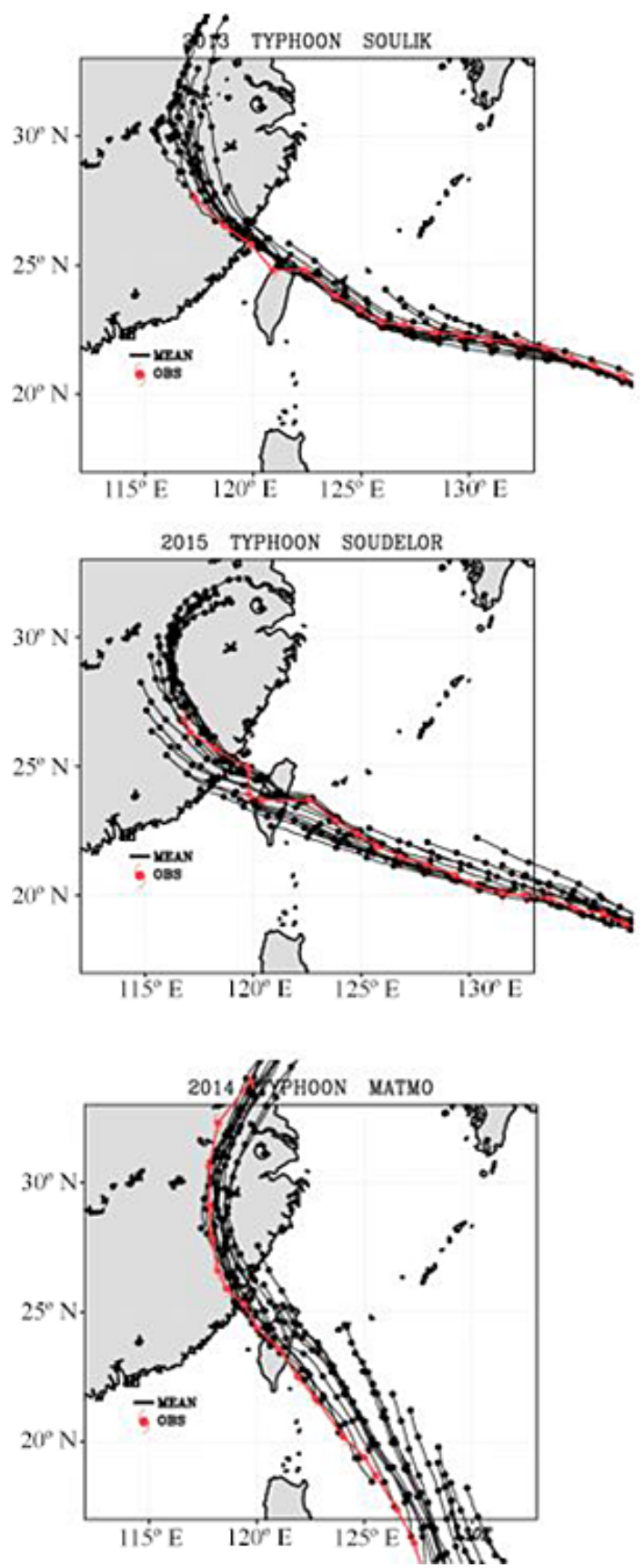

Figure 6. Comparisons of forecasted and observed typhoon tracks for Soulik (top), Soudelor (middle), and Matmo (bottom): black lines are TAPEX's ensemble mean forecasted tracks and each black line's forecasting length is $72 \mathrm{~h}$.

and $100 \%$ ) were selected. The results showed that forecasts with lower possibility thresholds had higher TSs (Figs. 7-9). For example, Fig. 7 shows that the TSs of Soudelor are 0.10.4 for the $10 \%$ probability threshold, which are higher than those for the $70 \%$ probability threshold. All tables showed that the average performance of low-possibility thresholds over the evaluation period resulted in better TS and POD 


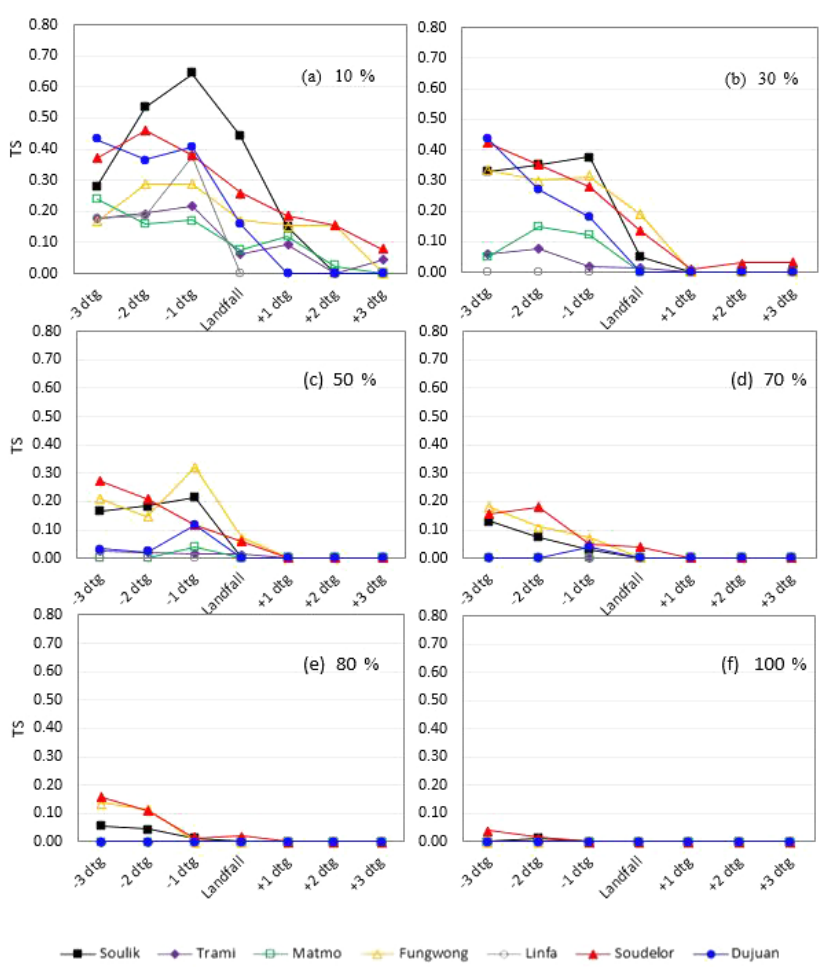

Figure 7. Comparisons of TS performance with a 1 to $24 \mathrm{~h}$ lead time considering various probability thresholds without a data assimilation technique.

scores. A lower probability threshold means a lower inundation threshold. Thus, the number of hits increased, but the number of false alarms increased as well. Decision makers generally consider an increased number of actions "in vain" when taking emergency measures based on a low probability threshold. The higher probability thresholds (e.g., a probability threshold $>50 \%$ ) had lower TSs and indicated that TAPEX ensemble rainfall forecasts were usually underestimated in this study. TAPEX's forecasted tracks had an impact on the rainfall forecasts, which affected the accuracy of the inundation forecasting. Soudelor and Soulik had the best performance in terms of TSs. The results for these typhoons were consistent with the track forecasts' performance (Fig. 5). The results also showed that the TS performance decreased after the typhoons made landfall. The period from $-3 \mathrm{dtg}$ to landfall is shown in Figs. 7-9. The steep terrain of Taiwan poses a challenge to the vortex initialization in numerical weather prediction models. Most current techniques are unable to properly initiate a typhoon vortex near complex terrain, when in reality the typhoons are already well developed at the time of landfall. The typhoons, due to their proximity to Taiwan by the time of model initiation, are not well developed in the models because of the terrain. The vortex is initialized near the complex terrain, and the current technique in TAPEX may not perform as well as it does when the vortex is in the open ocean. This introduces errors into the

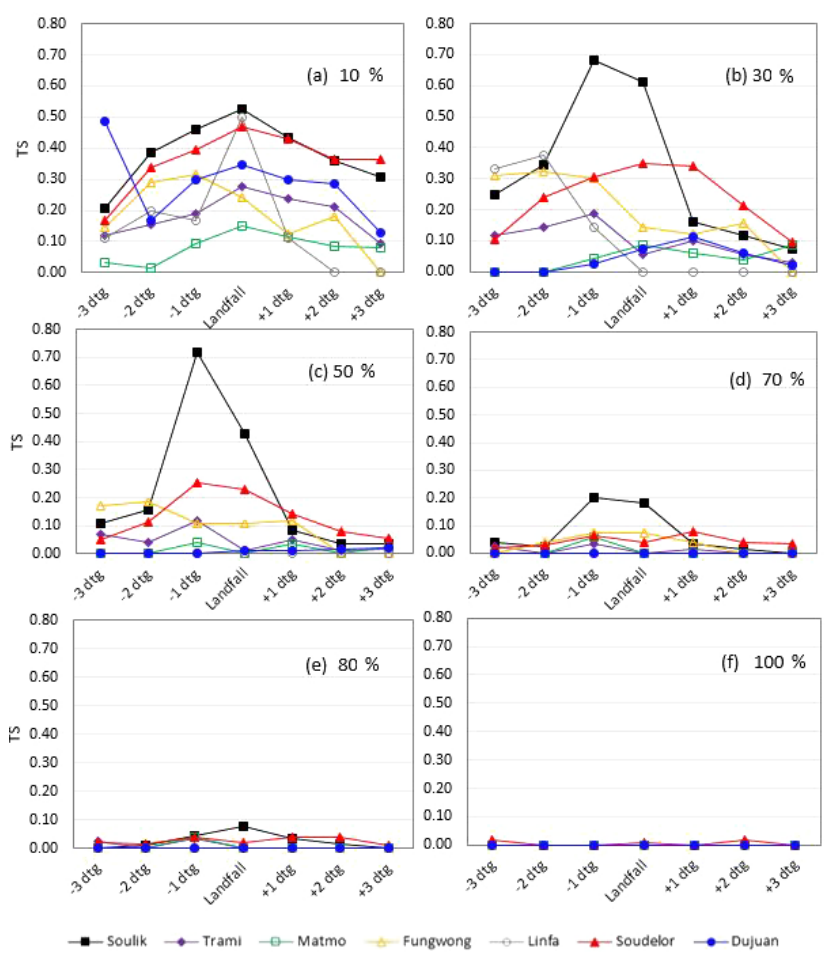

Figure 8. Comparisons of TS performance with a 25 to $48 \mathrm{~h}$ lead time considering various probability thresholds without a data assimilation technique.

consequent precipitation forecast. This observation explains the decreased system performance when the TAPEX model initialization involves a typhoon close to or making landfall on Taiwan, even if the forecast time is as small as 1 to $24 \mathrm{~h}$. The same issue does not create problems when the lead time is greater (or the typhoon is farther away). However, due to the complexity of the atmosphere, other issues, such as lack of observations, can cause the initial field degradation. Consequently, the typhoon tracks, rainfall, and related inundation forecasts were inevitably influenced. In the tables, the majority of ACC values exceeded 0.7. The less likely the inundation, the higher the ACC value. For example, only a few inundation alerts were issued during Linfa; the system's corresponding ACC scores were above 0.9. However, the POD and SR-FAR values were not as good as the ACC values in this case. The POD scores were zero. The SR-FAR values could not be calculated because there were zero hits and false alarms. When the system produced less accurate forecasts, the performance of the POD and SR-FAR functions decreased, resulting in a lower number of observed inundation alerts. A large number of inundation alerts were issued by the WRA during Soudelor and Soulik. The ACC numbers were below 0.8. The POD and SR-FAR numbers were relatively better than those in Linfa. A lower possibility threshold indicated that more hits and false alarms occurred; this resulted in negative SR-FAR scores. 


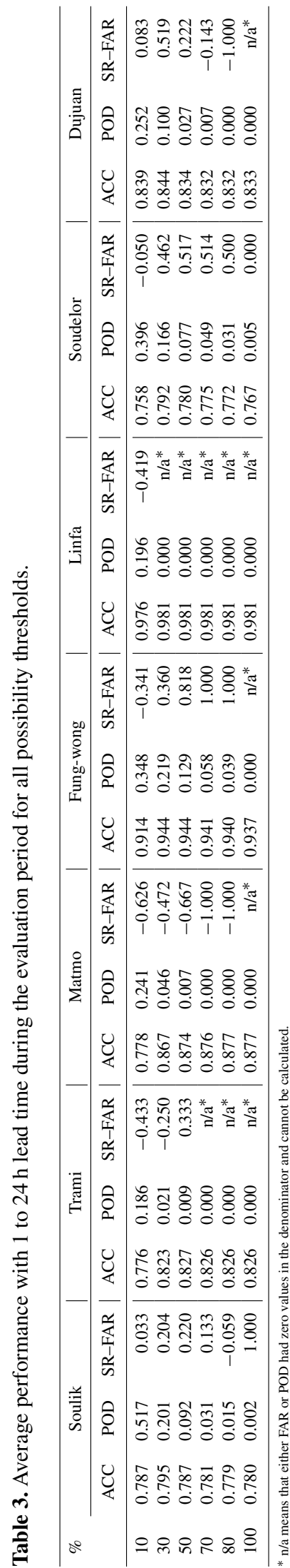

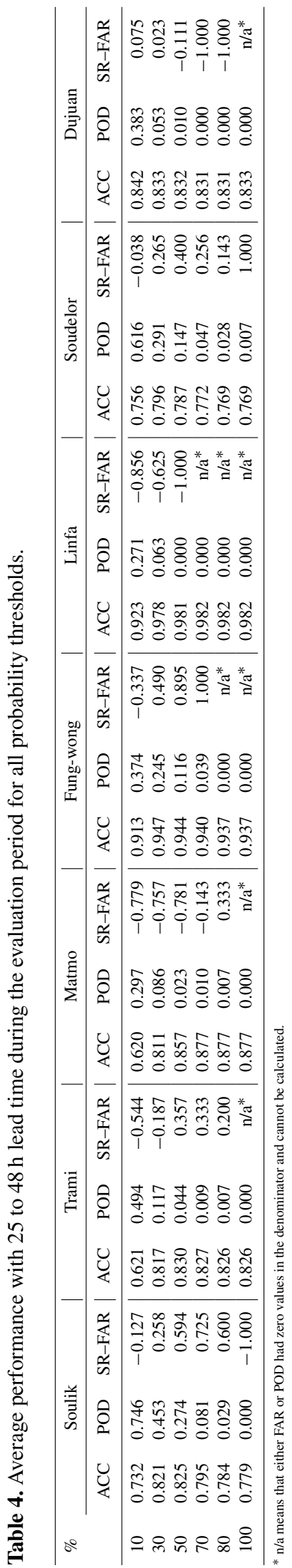

Hydrol. Earth Syst. Sci., 20, 4731-4745, 2016 


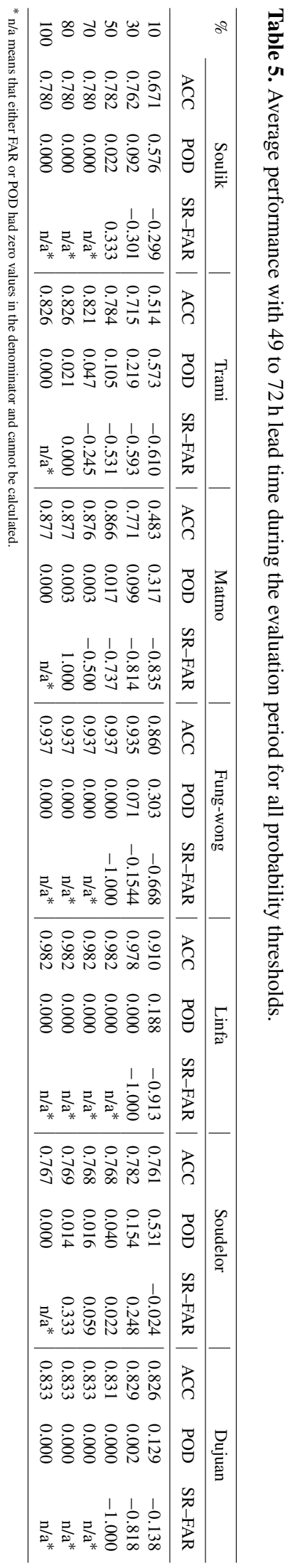

Hydrol. Earth Syst. Sci., 20, 4731-4745, 2016

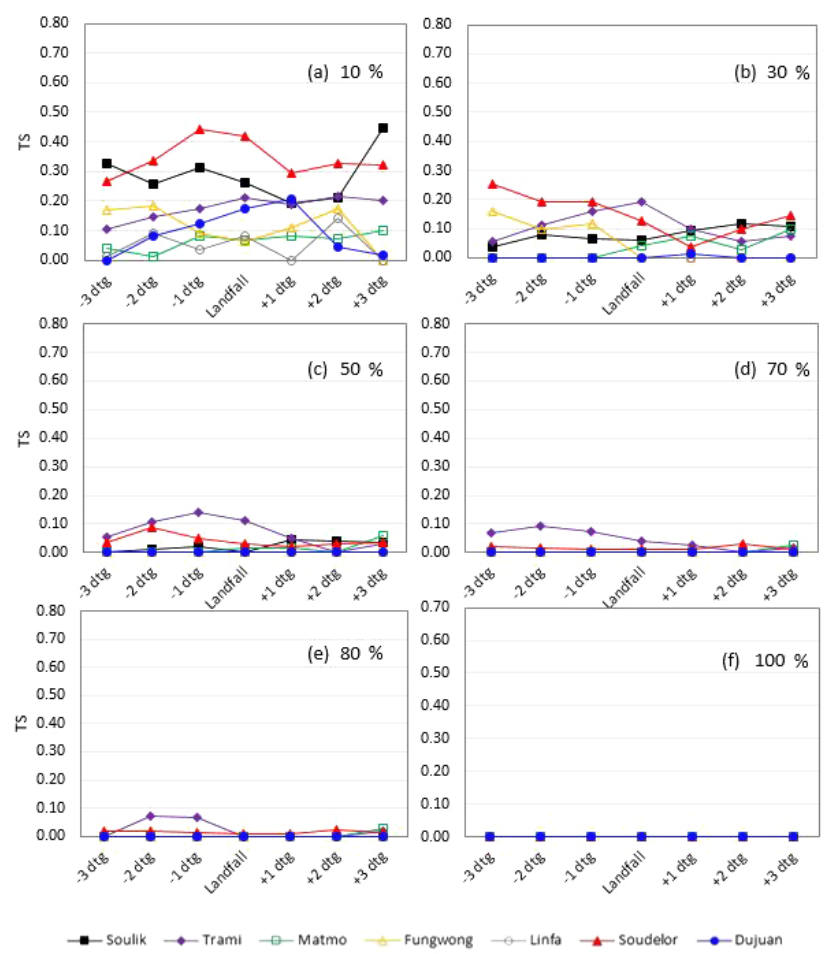

Figure 9. Comparisons of TS performance with a 49 to $72 \mathrm{~h}$ lead time considering various probability thresholds without a data assimilation technique.

In general, the SR-FAR scores decreased when the forecast lead time increased. However, the results for Soulik were opposite for the $50 \%$ probability threshold and below. The TS was higher when the probability increased by up to $50 \%$ prior to the typhoon making landfall (i.e., $-1 \mathrm{dtg}$ ). The number of false alarms decreased when the probability threshold increased. This helped improve the TS at $-1 \mathrm{dtg}$. However, this finding did not hold true when the probability threshold was above $70 \%$. Typhoon Matmo performed worst in terms of SR-FAR scores for the three different lead-time lengths. Figure 5 shows that the forecasted tracks did not coincide with the observed track. When a typhoon made landfall, the topography affected the performance of the numerical weather models, worsening the performance of the inundation warning forecasts. All of the results above indicate that the greatest uncertainty in the forecasts appears in the numerical weather predictions, which also has an important impact on other related disaster forecasts.

\subsection{Modified forecasts using the data assimilation technique}

To decrease the uncertainty of numerical weather predictions and improve the performance of inundation alert forecasting, this study applied a data assimilation technique that combined real-time observed and forecasted rainfall amounts to modify the forecasts. The data assimilation technique de- 


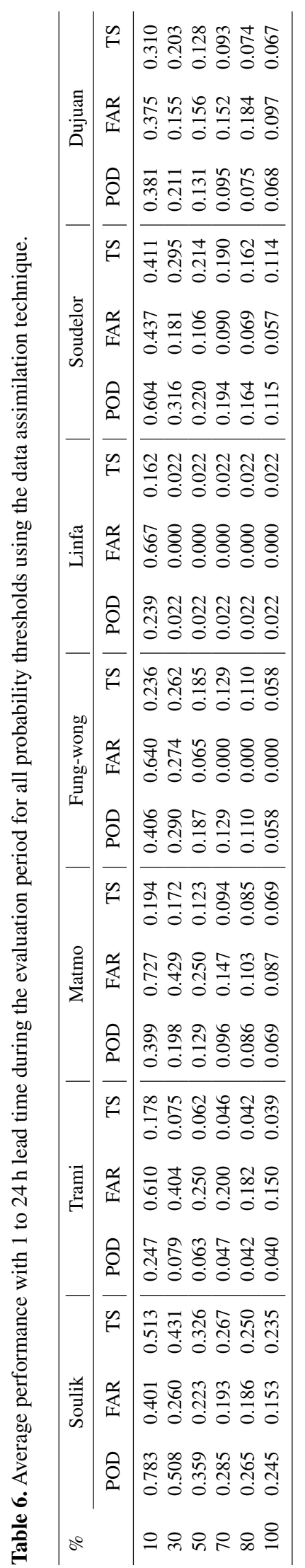

Table 7. Performance of all typhoons with the data assimilation technique for 1 to 24,25 to 48 , and 49 to $72 \mathrm{~h}$ lead time

\begin{tabular}{lcc|cc|cc}
\hline$\%$ & \multicolumn{2}{c|}{$1-24 \mathrm{~h}$} & \multicolumn{2}{c|}{$25-48 \mathrm{~h}$} & \multicolumn{2}{c}{$49-72 \mathrm{~h}$} \\
\cline { 2 - 7 } & FAR & TS & FAR & TS & FAR & TS \\
\hline 10 & 0.51 & 0.33 & 0.67 & 0.25 & 0.76 & 0.18 \\
30 & 0.26 & 0.25 & 0.49 & 0.19 & 0.75 & 0.08 \\
50 & 0.18 & 0.18 & 0.34 & 0.11 & 0.72 & 0.03 \\
70 & 0.15 & 0.15 & 0.29 & 0.03 & 0.59 & 0.01 \\
80 & 0.14 & 0.13 & 0.38 & 0.01 & 0.42 & 0.01 \\
100 & 0.12 & 0.11 & 0.20 & 0.00 & - & 0.00 \\
\hline
\end{tabular}

creased the temporal uncertainty of numerical rainfall forecasts and improved the accuracy of early warning notifications. The longest rainfall threshold duration to trigger an inundation alerts is $24 \mathrm{~h}$ in this study. The technique was used to address the gap in forecasted rainfall data with observed rainfall information. The absence of forecasted rainfall values occurred in the first warning period (i.e., 1-24h). Therefore, this study used the data assimilation technique to improve the 1 to $24 \mathrm{~h}$ forecasts. Table 6 shows the modified forecast results compared to the original forecasts. Compared to the results without the hybrid technique, all performance measures' scores improved significantly. For example, when all typhoons were tested using the original forecasts, the system performed best during Soulik. Using the hybrid technique, the POD scores improved from 0.517 to 0.783 and from 0.002 to 0.245 for the 10 and $100 \%$ probability thresholds, respectively. The TSs improved from 0.293 to 0.513 and from 0.002 to 0.235 for the 10 and $100 \%$ probability thresholds, respectively. The probability threshold represents the number of ensemble members' forecasted rainfall events that met or exceeded the rainfall thresholds. The hybrid technique forecasts thus support the idea that a higher probability threshold indicates lower uncertainty in terms of forecasting. The FAR and POD scores decreased when the probability threshold increased. Decision-making confidence increases when the probability threshold increases and the FAR decreases. Coughlan de Perez et al. (2016) concluded that the likelihood of taking a necessary action when the FAR is lower than 0.5 would satisfy the decision maker's requirements for not taking action potentially in vain. Table 6 shows that most of the FAR scores improved to below 0.5 using the hybrid technique. Though these values improved compared to previous results, all of the POD scores were still low and continued to decrease when the probability threshold increased. The low POD score implies a lower hit rate. To improve these values, identifying the accuracy and uncertainty of rainfall forecasts is necessary.

Table 7 shows the overall performance of the system for seven typhoons in terms of FAR and TS scores. The overall results indicate that the FAR score decreases when the possibility threshold increases. The FAR score is smaller than 
0.5 when the possibility is greater than $30 \%$ with a lead time of $48 \mathrm{~h}$. Therefore, the system performance meets the requirements of decision makers to take action during typhoon events (Coughlan de Perez et al., 2016). However, the system cannot provide acceptable forecasts with a lead time greater than $48 \mathrm{~h}$, regardless of which possibility threshold is selected. This finding limits the use of the system when the lead time is greater than $48 \mathrm{~h}$. The system integrates TAPEX data to obtain forecasted typhoon tracks and rainfall amounts. However, for some local convections, such as afternoon thunderstorms, the current $5 \mathrm{~km}$ spatial resolution of TAPEX might not be sufficient to resolve these weather phenomena as well as it does for much larger-scale weather systems, such as typhoons. These small-scale weather systems pose another limitation to the use of this system.

\section{Conclusions}

This study proposed an early inundation warning system that integrates ensemble rainfall forecasts and rainfall thresholds. Five rainfall thresholds with different durations were applied. Seven typhoon events during the period 2013-2015 and real inundation alert records from the WRA were used to evaluate the performance of the system. Five performance measures and a period of $18 \mathrm{~h}$ ( $3 \mathrm{dtg}$ 's) before and after a typhoon made landfall were considered. The system applied ensemble rainfall forecasts and provided probabilistic forecasts. Therefore, six different probability thresholds were considered to trigger the issuance of inundation alerts and calculate various performance scores. An appropriate probability threshold helps decision makers take fewer actions in vain. The results showed that a lower probability threshold had a higher POD score, which is associated with a higher inundation alert detection rate. The downside of a lower probability threshold is a higher FAR score. If the FAR is above 0.5, the system is considered impractical (Coughlan de Perez et al., 2016). Although the system performed better before a typhoon made landfall, particularly in terms of TSs, it was still unable to identify the most useful probability threshold for identifying when emergency responders should take various actions. $\mathrm{Nu}-$ merical weather predictions were the dominant input influencing the forecast results. The system's performance varied according to the different typhoons tested. In other words, the system cannot maintain a constant level of performance due the temporal and spatial uncertainties in the numerical rainfall forecasts. Taiwan's steep terrain also poses a challenge to the vortex initialization in numerical weather prediction models and contributes to the uncertainty inherent in the rainfall forecasts. In conclusion, the findings of this study suggest that a better forecast is usually produced (1) when the forecasted typhoon tracks are consistent with the observed tracks and (2) before a typhoon makes landfall.
Finally, the authors developed a data assimilation technique that combined real-time observed and forecasted rainfall to decrease the uncertainty of numerical weather predictions and to improve $24 \mathrm{~h}$ inundation forecasts. The results showed that the FAR scores decreased when the probability threshold increased. All FAR scores were below 0.5 or less when the probability threshold was $30 \%$ or above. This technique improved the appeal of the early warning system and generated more valuable forecasts that allowed decision makers to take fewer actions in vain. To further decrease the uncertainty of numerical weather predictions and improve the performance of inundation forecasts, advanced techniques, such as radar observations and associated data assimilation systems, could be considered in the future. A greater number of extreme weather events are likely in the future due to global climate change. These extreme events will bring high-intensity rainfalls over very short time spans. Radar observations efficiently improve very short-range rainfall forecasts, which are essential for accurate inundation forecasts. Rainfall thresholds need to be updated to meet the present flood capacity, such as when a new storm sewage system is put in place. After all, decision makers use forecasted rainfall and threshold-based early warning systems for a high-level overview of flood risk only. Given its advantage of an extended lead time and rapid estimation process, the model presented here is beneficial for emergency deployment to prepare large areas in advance of flooding. For small-area forecasts during a disaster, a complex physics-based model is recommended to replace the threshold-based model and provide detailed information.

\section{Data availability}

The TAPEX's rainfall forecast data were provided by the Taiwan Typhoon and Flood Research Institute (TTFRI, 2016). The government's law prohibits the data to be freely distributed. An application is needed before receiving the raw data. The rainfall thresholds for all townships were identified by the Water Resources Agency in Taiwan (WRA, 2016). National Science and Technology Center for Disaster Reduction in Taiwan provided the historical flood warnings of various typhoons (NCDR, 2016). 


\section{Appendix A: List of abbreviations}

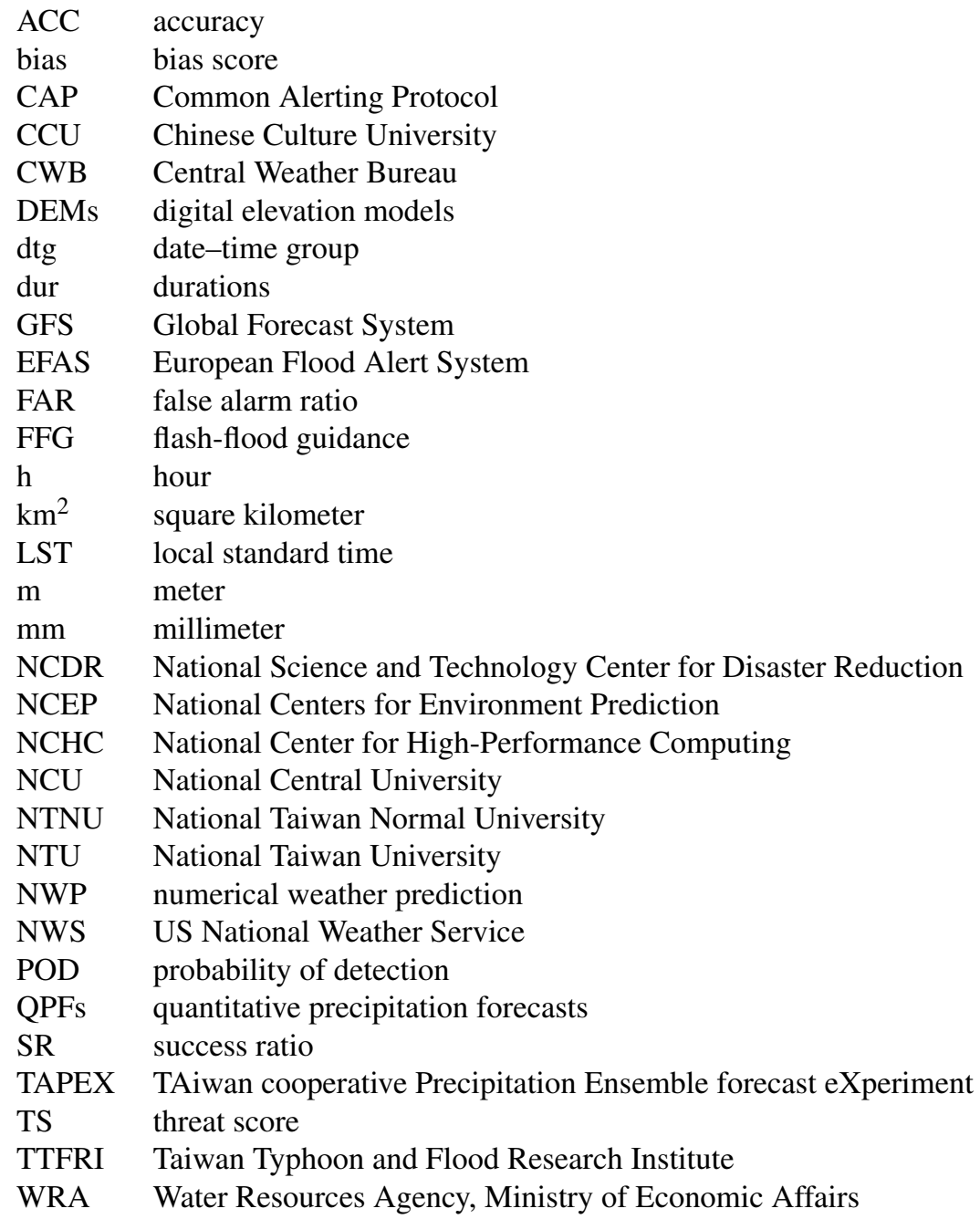


Acknowledgements. The authors would like to thank the Water Resources Agency, Taiwan, for providing the rainfall threshold values and the Central Weather Bureau, Taiwan, for providing rainfall observation data.

Edited by: H. Cloke

Reviewed by: two anonymous referees

\section{References}

Alfieri, L., Salamon, P., Pappenberger, F., Wetterhall, F., and Thielen, J.: Operational early warning systems for water-related hazards in Europe, Environ. Sci. Policy, 21, 35-49, 2012.

Alfieri, L., Pappenberger, F., and Wetterhall, F.: The extreme runoff index for flood early warning in Europe, Nat. Hazards Earth Syst. Sci., 14, 1505-1515, doi:10.5194/nhess-14-1505-2014, 2014.

Badrzadeh, H., Sarukkalige, R., and Jayawardena, A. W.: Hourly runoff forecasting for flood risk management: application of various computational intelligence models, J. Hydrol., 529, 16331643, 2015.

Barredo, J. I.: Normalised flood losses in Europe: 1970-2006, Nat. Hazards Earth Syst. Sci., 9, 97-104, doi:10.5194/nhess-9-972009, 2009.

Carpenter, T. M., Sperfslage, J. A., Georgakakos, K. P., Sweeney, T., and Fread, D. L.: National threshold runoff estimation utilizing GIS in support of operational flash flood warning systems, J. Hydrol., 224, 21-44, 1999.

Chang, L., Shen, H., Wang, Y., Huang, J., and Lin, Y.: Clusteringbased hybrid inundation model for forecasting flood inundation depths, J. Hydrol., 385, 257-268, 2010.

Cheng, C. and Liao, W.: Current situation and sustainability of water resource in Taiwan, in: Proceedings of 1st Asian Water Saving Council Conference, 141-148, 2011.

Cirbus, J. and Podhoranyi, M.: Cellular automata for the flow simulations on the earth surface, optimization computation process, Appl. Math. Inf. Sci., 7, 2149-2158, 2013.

Cloke, H. L. and Pappenberger, F.: Ensemble flood forecasting: a review, J. Hydrol., 375, 613-626, 2009.

Coughlan de Perez, E., van den Hurk, B., van Aalst, M. K., Jongman, B., Klose, T., and Suarez, P.: Forecast-based financing: an approach for catalyzing humanitarian action based on extreme weather and climate forecasts, Nat. Hazards Earth Syst. Sci., 15, 895-904, doi:10.5194/nhess-15-895-2015, 2015.

Coughlan de Perez, E., van den Hurk, B., van Aalst, M. K., Amuron, I., Bamanya, D., Hauser, T., Jongma, B., Lopez, A., Mason, S., Mendler de Suarez, J., Pappenberger, F., Rueth, A., Stephens, E., Suarez, P., Wagemaker, J., and Zsoter, E.: Actionbased flood forecasting for triggering humanitarian action, Hydrol. Earth Syst. Sci., 20, 3549-3560, doi:10.5194/hess-20-35492016, 2016.

Crosta, G. B. and Frattini, P.: Distributed modelling of shallow landslides triggered by intense rainfall, Nat. Hazards Earth Syst. Sci., 3, 81-93, doi:10.5194/nhess-3-81-2003, 2003.

Dale, M., Wicks, J., Mylne, K., Pappenberger, F., Laeger, S., and Taylor, S.: Probabilistic flood forecasting and decision-making: an innovative risk-based approach, Nat. Hazards, 70, 159-172, 2014.
De Kleermaeker, S., Verlaan, M., Kroos, J., and Zijl, F.: A new coastal flood forecasting system for the Netherlands, Proceedings of Hydro 2012 "Taking Care of the Sea", Rotterdam, the Netherlands, 13-15 November 2012, 135-140, 2012.

Doong, D.-J., Chuang, L. Z.-H., Wu, L.-C., Fan, Y.-M., Kao, C. C., and Wang, J.-H.: Development of an operational coastal flooding early warning system, Nat. Hazards Earth Syst. Sci., 12, 379390, doi:10.5194/nhess-12-379-2012, 2012.

Georgakakos, K.: Modern operational flash flood warning systems based on flash flood guidance theory: performance evaluation, International Conference on Innovation Advances and Implementation of Flood Forecasting Technology, Bergen-Troms $\varnothing$, Norway, 9-13 October 2005, 1-10, 2005.

Georgakakos, K. P.: Analytical results for operational flash flood guidance, J. Hydrol., 317, 81-103, 2006.

Georgakakos, K., Graham, R., Jubach, R., Modrick, T., Shamir, E., Spencer, C., and Sperfslage, J.: Global flash flood guidance system, phase I, Hydrological Research Center, San Diego, CA, HRC Technical Report No. 9, 2013.

Gourley, J. J., Flamig, Z. L., Hong, Y., and Howard, K. W.: Evaluation of past, present and future tools for radar-based flash-flood prediction in the USA, Hydrol. Sci. J., 59, 1377-1389, 2014.

Gruntfest, E. and Handmer, J. (Eds.): Coping with Flash Floods, NATO Science Series, Vol. 77, Springer Verlag Science, Dordrecht, the Netherlands, 243 pp., 2001.

Guzzetti, F., Peruccacci, S., Rossi, M., and Stark, C. P.: Rainfall thresholds for the initiation of landslides in central and southern Europe, Meteorol. Atmos. Phys., 98, 239-267, 2007.

Hallegatte, S.: A cost effective solution to reduce disaster losses in developing countries: hydro-meteorological services, early warning, and evacuation, World Bank Policy Research Working Paper 6058, 2012.

Hallegatte, S., Green, C., Nicholls, R. J., and Corfee-Morlot, J.: Future flood losses in major coastal cities, Nat. Clim. Change, 3, 802-806, 2013.

Hirabayashi, Y., Mahendran, R., Koirala, S., Konoshima, L., Yamazaki, D., Watanabe, S., Kim, H., and Kanae, S.: Global flood risk under climate change, Nat. Clim. Change, 3, 816-821, 2013.

Hoedjes, J., Kooiman, A., Maathuis, B., Said, M., Becht, R., Limo, A., and Su, B.: A conceptual flash flood early warning system for Africa based on terrestrial microwave links and flash flood guidance, ISPRS Int. J. Geoinf., 3, 584-598, 2014.

Hsiao, L., Chen, D., Kuo, Y., Guo, Y., Yeh, T., Hong, J., Fong, C., and Lee, C.: Application of WRF 3DVAR to operational typhoon prediction in Taiwan: impact of outer loop and partial cycling approaches, Weather Forecast., 27, 1249-1263, 2012.

Hsiao, L., Yang, M., Lee, C., Kuo, H., Shih, D., Tsai, C., Wang, C., Chang, L., Chen, D. Y., Feng, L., Hong, J., Fong, C., Chen, D., Yeh, T., Huang, C., Guo, W., and Lin, G.: Ensemble forecasting of typhoon rainfall and floods over a mountainous watershed in Taiwan, J. Hydrol., 506, 55-68, 2013.

Huang, J., Yu, C., Lee, J., Cheng, L., Lee, T., and Kao, S.: Linking typhoon tracks and spatial rainfall patterns for improving flood lead time predictions over a mesoscale mountainous watershed, Water Resour. Res., 48, W09540, doi:10.1029/2011WR011508, 2012.

Huthoff, F., Remo, J. W. F., and Pinter, N.: Improving flood preparedness using hydrodynamic levee-breach and inundation 
modelling: middle Mississippi River, USA, J. Flood Risk Manage., 8, 2-18, 2015.

Jang, J.: An advanced method to apply multiple rainfall thresholds for urban flood warnings, Water, 7, 6056-6078, 2015.

Jang, J., Yu, P., Yeh, S., Fu, J., and Huang, C.: A probabilistic model for realtime flood warning based on deterministic flood inundation mapping, Hydrol. Process., 26, 1079-1089, 2012.

Lee, C., Wu, S., and Chang, T.: Implementing common alerting protocol for disaster reduction in Taiwan, 2014 TGIS GeoInformatics - UGIS Joint International Conference, Kaohsiung, Taiwan, 25-27 June, 2014.

Lin, G. and Jhong, B.: A real-time forecasting model for the spatial distribution of typhoon rainfall, J. Hydrol., 521, 302-313, 2015.

Lin, G., Lin, H., and Chou, Y.: Development of a real-time regionalinundation forecasting model for the inundation warning system, J. Hydroinform., 15, 1391-1407, 2013.

Liu, L., Liu, Y., Wang, X., Yu, D., Liu, K., Huang, H., and Hu, G.: Developing an effective 2-D urban flood inundation model for city emergency management based on cellular automata, Nat. Hazards Earth Syst. Sci., 15, 381-391, doi:10.5194/nhess-15381-2015, 2015.

López-Trujillo, D.: Real Time Flood Alert System (RTFAS) for Puerto Rico, US Department of Interior, US Geological Survey, 2010.

Martina, M. L. V., Todini, E., and Libralon, A.: A Bayesian decision approach to rainfall thresholds based flood warning, Hydrol. Earth Syst. Sci., 10, 413-426, doi:10.5194/hess-10-413-2006, 2006.

McBride, J. L. and Ebert, E. E.: Verification of quantitative precipitation forecasts from operational numerical weather prediction models over Australia, Weather Forecast., 15, 103-121, 2000.

National Science and Technology Center for Disaster Reduction, Taiwan (NCDR): Observed flood warnings for typhoons from 2013 to 2015, retrieved from: https://alerts.ncdr.nat.gov.tw/ AlertdataDownload.aspx, 2016.

Nguyen, P., Thorstensen, A., Sorooshian, S., Hsu, K., and AghaKouchak, A.: Flood forecasting and inundation mapping using HiResFlood-UCI and near-real-time satellite precipitation data: the 2008 Iowa flood, J. Hydrometeorol., 16, 1171-1183, 2015.

OASIS Emergency Management Technical Committee: Common alerting protocol, v.1.1., Organization for the Advancement of Structured Information Standards (OASIS), Boston, MA, 2005.

Pappenberger, F., Beven, K. J., Hunter, N. M., Bates, P. D., Gouweleeuw, B. T., Thielen, J., and de Roo, A. P. J.: Cascading model uncertainty from medium range weather forecasts $(10$ days) through a rainfall-runoff model to flood inundation predictions within the European Flood Forecasting System (EFFS), Hydrol. Earth Syst. Sci., 9, 381-393, doi:10.5194/hess-9-381-2005, 2005.

Posner, A. J. and Georgakakos, K. P.: Soil moisture and precipitation thresholds for real-time landslide prediction in El Salvador, Landslides, 12, 1179-1196, 2015.
Raynaud, D., Thielen, J., Salamon, P., Burek, P., Anquetin, S., and Alfieri, L.: A dynamic runoff co-efficient to improve flash flood early warning in Europe: evaluation on the 2013 central European floods in Germany, Meteorol. Appl., 22, 410-418, 2015.

Sampson, C. C., Smith, A. M., Bates, P. D., Neal, J. C., Alfieri, L., and Freer, J. E.: A high-resolution global flood hazard model, Water Resour. Res., 51, 7358-7381, 2015.

Shamir, E., Georgakakos, K. P., Spencer, C., Modrick, T. M., Murphy Jr., M. J., and Jubach, R.: Evaluation of real-time flash flood forecasts for Haiti during the passage of hurricane Tomas, 4-6 November, 2010, Nat. Hazards, 67, 459-482, 2013.

Shao, Q., Weatherley, D., Huang, L., and Baumgartl, T.: RunCA: a cellular automata model for simulating surface runoff at different scales, J. Hydrol., 529, 816-829, 2015.

Shi, H., Li, T., Liu, R., Chen, J., Li, J., Zhang, A., and Wang, G.: A service-oriented architecture for ensemble flood forecast from numerical weather prediction, J. Hydrol., 527, 933-942, 2015.

Taiwan Typhoon and Flood Research Institute (TTFRI): TAPEX's rainfall forecasts for typhoons during 2013 to 2015, retrieved from: https://tf-rat.narlabs.org.tw/member/login/?next=/, 2016.

Thielen, J., Bartholmes, J., Ramos, M.-H., and de Roo, A.: The European Flood Alert System - Part 1: Concept and development, Hydrol. Earth Syst. Sci., 13, 125-140, doi:10.5194/hess-13-1252009, 2009.

Water Resources Agency, Ministry of Economic Affairs, Taiwan (WRA): Rainfall thresholds for townships in Taiwan, retrieved from: http://fhy.wra.gov.tw/Pub_Web_2011/Page/ SurveyStationInfos.aspx, 2016.

Wilks, D.: Statistical Methods in the Atmospheric Sciences, 2nd Edn. Elsevier, Burlington, 2005.

Wu, D.: Rainfall threshold values for flood inundation, Workshop on Flood and Drought Prevention and Mitigation, Taipei, Taiwan, 12 April 2012, 2013.

Wu, D. and Wang, Y.: A research on using critical precipitation value for taiwan inundation warning system, in: Proceedings of the 2009 Conference for Disaster Management in Taiwan, Taipei, Taiwan, 19 December, 2009.

Wu, S., Hsu, C., Lien, H., and Chang, C.: Modeling the effect of uncertainties in rainfall characteristics on flash flood warning based on rainfall thresholds, Nat. Hazards, 75, 1677-1711, 2015.

Yang, T., Yang, S., Ho, J., Lin, G., Hwang, G., and Lee, C.: Flash flood warnings using the ensemble precipitation forecasting technique: a case study on forecasting floods in Taiwan caused by typhoons, J. Hydrol., 520, 367-378, 2015.

Zhang, Y., Reed, S., Gourley, J. J., Cosgrove, B., Kitzmiller, D., Seo, D., and Cifelli, R.: The impacts of climatological adjustment of quantitative precipitation estimates on the accuracy of flash flood detection, J. Hydrol., 541, 387-400, 2015. 